\title{
HEAVY PARTICLES AND THE $\rho$ PARAMETER IN THE STANDARD MODEL
}

\author{
M.B. EINHOR.N \\ Linicersity of Michigan. Randall Laboraton of Phisics*. Ann Arbor, MI 48109 \\ and \\ Institute of Theorencal Phisics, Linicersity of Califormia, Sama Barhara. CA 93106. USA
}

D.R.T. JONES

University of Michigan, Randall Laboratory of Phisics, Ann Arbor. MI 48109, LSA

M. VELTMAN

Institute for Theoretical Phisics Utrecht. The Netherlands

and

Unitersity of Michigan, Randall Laboratony of Phisics *. Ann Arbor, MI 48109. LSA

Received 5 May 1981

\begin{abstract}
A new formalism is presented for the calculation of the contribution $\delta \rho$ to $\rho=M_{\mathrm{W}}^{2} / M_{\mathrm{Z}}^{2} \cos ^{2} \theta_{\mathrm{W}}$ from heavy particles transforming according to arbitrary representations of $\mathrm{SU}(2) \times \mathrm{U}(1)$. A conjecture for the necessary and sufficient conditions that $\delta \rho \geqslant 0$ for all values of masses and mixing angles within a particular multiplet is formulated. A number of examples are given (all consistent with the conjecture) and the significance of improved knowledge of $\rho$ vis à vis the possible existence of undiscovered heavy particles discussed.
\end{abstract}

\section{Introduction}

The standard $\mathrm{SU}(2) \times \mathrm{U}(1)$ model provides an increasingly successful description of low-energy weak and electromagnetic processes. Its theoretical framework, however, places few restrictions on the number and type of undiscovered particles. It is therefore important to ask whether information about heavy particles can be obtained by measurements at currently accessible energies. In this paper we focus attention on the measurement of the parameter $\rho=M_{\mathrm{W}}^{2} / M_{\mathrm{Z}}^{2} \cos ^{2} \theta_{\mathrm{W}}$.

In the standard model, $\rho=1$ in the tree approximation, a consequence of an "accidental" SU(2) symmetry of the vector boson mass matrix [1]. The fact that $\rho \approx 1$ experimentally ${ }^{\star}$ provides strong support for the doublet Higgs structure. Deviations of $\rho$ from one are expected due to radiative corrections, among which are the vacuum polarization of the $\mathrm{W}$ and $\mathrm{Z}$ propagators. As emphasized previously

* Address after September 1. 1981.

ॠ See, for example, the recent fits of ref. [2]. 
$[3,7]$, these are especially sensitive to heavy particles in the range of $100 \mathrm{GeV}$. As experiments improve the precision with which $\rho$ is known, insight may be gained into as yet undiscovered heavy particles.

Of course, radiative corrections are energy dependent and, among other effects, cause shifts in the theoretically expected values of the physical masses $M_{\mathrm{W}}$ and $M_{\mathrm{Z}}$ [4]. In this paper, we consider contributions to the vacuum polarization due to heavy particles and energies well below the masses of such hypothetical particles. Our investigation is motivated by the observation that, for standard lepton or quark families, the correction $\delta \rho=\rho-1$ satisfies $\delta \rho \geqslant 0$ for all values of the fermion masses, so that additional generations seem to always increase $\rho$. Although these corrections have been calculated for a number of special cases [3-7] no general or systematic study has been made, and this provides further motivation for our work.

In the following, we consider one-loop corrections to the vector boson propagators due to heavy fermions and/or scalars (which do not develop vacuum expectation values). For $q^{2} \ll M_{\mathrm{W}}^{2}$ and also well below the heavy particle masses, the polarization tensor $\Pi_{a b}^{\mu \nu}\left(q^{2}\right)$ is essentially constant and may be approximated by its value at zero,

$$
\Pi_{a b}^{\mu \nu}(0)=g^{\mu \nu} \Pi_{a b}(0) .
$$

(Here $a, b$ denote weak isospin indices.) We are especially interested in the difference $\delta \Pi$ between the contributions to the $\mathrm{W}$ and $\mathrm{Z}$ :

$$
\delta \rho=\Pi_{+-} / M_{\mathrm{W}}^{2}-\Pi_{\mathrm{ZZ}} / M_{\mathrm{Z}}^{2}=\delta \Pi 1 / M_{\mathrm{W}}^{2} .
$$

At $q^{2}=0$, this is

$$
\delta \Pi=\Pi_{+}-\Pi_{33} .
$$

We present a conjecture that, for a certain general class of contributions, $\delta \Pi(0) \geqslant 0$ for all values of the masses of the associated multiplet ${ }^{\star \star}$. We show that the conjectured result holds for a number of special cases falling within the class but have been unable to prove it in full generality. We also exhibit models, both for scalars and for fermions, which fall outside the class and can give contributions to $\delta \rho$ of either sign. As a by product of this work, we derive a compact expression for $\delta \mathrm{II}$ which simplifies the calculation in any given model and provides a more convenient form for discussing positivity and symmetry properties.

\footnotetext{
* In general, there are corrections of order $\alpha q^{2}$ to $\delta \Pi$. Also. $\delta \rho$ receives additional corrections, such as $\gamma Z$ interference or other, process-dependent, corrections. See refs. [6,8]. A further complication is that different authors use different definitions of the weak mixing angle: for example, in ref. [6], $\rho$ is defined to be 1. so our calculation would be interpreted as a correction to the tree relation $M_{\mathrm{w}}=\left(\pi \alpha / \sqrt{2} G_{\mu}\right)^{1 / 2} / \sin \theta_{\mathrm{W}}$. We choose to follow ref. [8].

* The assertion that $\delta I I(0) \geqslant 0$ would follow if one could show that the imaginary part $\operatorname{Im} \delta \operatorname{II}\left(q^{2}\right) \geqslant 0$ for all $q^{2}$, since $\delta I I\left(q^{2}\right)$ obeys an unsubtracted dispersion relation. It is easy to see. however, that this stronger result is not true. Even for a simple standard lepton or quark doublet, with doublet masses $m_{1}$ and $m_{2}\left(m_{1}<m_{2}\right.$, say), there is a range $4 m_{1}^{2} \leqslant q^{2} \leqslant\left(m_{1}+m_{2}\right)^{2}$. where $\operatorname{ImII}, \quad\left(q^{2}\right)=0$ but $\operatorname{ImI} I_{77}\left(q^{2}\right) \geqslant 0$, so $\operatorname{Im} \delta I I \leqslant 0$. Nevertheless, $\delta I I(0) \geqslant 0$ (see sect. 3$)$.
} 
Before proceeding let us consider the experimental relevance of a non-vanishing $\delta \rho$. For a fermion multiplet whose mass $m$ arises entirely from symmetry breaking, the order of magnitude of $\delta \rho$ is $G_{\mathrm{F}} m^{2} / 8 \pi^{2}$. Now if we assume that experiments will eventually be sensitive to $\delta \rho \gtrsim 0.5 \%$, we find $m \gtrsim 200 \mathrm{GeV}$ for the effect to be observable. This is, however, near the value at which we expect lowest order perturbation theory to cease being trustworthy. A naive estimate based on the size of the Yukawa coupling of the fermion gives $m \lesssim 400 \mathrm{GeV}$. By considering partial-wave amplitude unitarity Chanowitz et al. [7] found $m \leqq 500 / \sqrt{ } N \mathrm{GeV}$. (1 TeV $/ \sqrt{ } N$ ) for $N$ nearly degenerate isodoublets of quarks (leptons). Thus the range of values for which our calculation is reliable is at best a narrow one. However, when perturbation theory breaks down because certain couplings become strong, then $\rho=1$ is no longer natural unless the strong interactions obey an isospin symmetry. Thus the simultaneous occurrence of $\rho \simeq 1$ and strong interactions among heavy particles places interesting restrictions on higher symmetries, as has been noted in technicolor models $[1,9]$.

We now turn briefly to a discussion of the problem of decoupling of heavy masses and its relevance to our calculations. We will discuss the decoupling issue in the context of the $\rho$ parameter but will attempt to make clear which conclusions have general validity. With regard to decoupling, two distinct cases arise in general. If the particles are in representations such that their masses arise entirely from spontaneous symmetry breakdown, then the contribution to $\rho$ is not suppressed as the masses are scaled up. However, since increasing the masses involves increasing a dimensionless coupling constant, masses cannot be made arbitrarily large without creating a sector of the theory that is strongly coupled and thereby invalidating perturbative calculations, as already discussed. If, on the other hand, the representation allows an $S U(2) \times U(1)$ symmetric mass term, then the associated particles can be made arbitrarily heavy, but it is found that the contributions to $\rho$ are now suppressed in accordance with the decoupling theorem [10]. The way this occurs is slightly different for scalar and fermion contributions. For scalars, when. of course, a symmetric mass term is always allowed. the natural order of magnitude of the mass difference $\Delta M$ is given by $\Delta M \sim g\langle\phi\rangle^{2} / M$, where $M$ is the heavy mass. $\langle\phi\rangle$ the vacuum expectation value of the Higgs and $g$ some dimensionless coupling. The contributions to $\rho$ are $\mathrm{O}\left(G_{\mathrm{F}}(\Delta M)^{2}\right)$ and one sees that the decoupling theorem holds. For fermions, we have $\Delta M \sim g\langle\phi\rangle$ and hence apparently no decoupling; and indeed that is what occurs in the first case considered above. However, in the case where an isospin invariant mass term $M$ is allowed, and we make $M \gg \Delta M$ one finds ${ }^{\star}$ that the contribution to $\rho$ is $\mathrm{O}\left(G_{\mathrm{F}}(\Delta M)^{4} / M^{2}\right)$; in other words the decoupling theorem is valid.

Now when the heavy mass scale is a result of a symmetry breaking at some higher energy it is clear that the heavy particles will lie in representations of $\operatorname{SU}(2) \times U(1)$

\footnotetext{
* We have explicitly verified the above statement for the case of a left-right symmetric model (see sect. 4). We believe. however, that the conclusion is quite general.
} 
degenerate except for the splittings induced by $\langle\phi\rangle$. This corresponds to the second case considered above, and decoupling occurs; so that, for example, in the SU(5) grand unified theory the contributions of the $\mathrm{X}$ and $\mathrm{Y}$ gauge bosons, and of any fermions which receive masses from the vacuum expectation value of the Higgs 24-plet, will be suppressed. For a more detailed discussion of the decoupling theorem and the related problem of gauge hierarchies in the context of grand unified theories, see refs. [11].

As noted earlier, $\rho=1$ at the tree level because of an accidental isospin symmetry of the spontaneously broken theory in the tree approximation [1] ${ }^{\star}$. In fact, $\rho=1$ if isospin were exactly conserved, but it is intrinsically broken by hypercharge interactions. Nevertheless, it remains useful to discuss the transformation properties of the theory under this isospin and to assign isospin transformation properties to all the fields. (This will be discussed in the next section.) At the one-loop level, non-vanishing contributions to $\delta \Pi$ arise from mass splittings within isomultiplets which are related to couplings in the original lagrangian. By considering a number of different fermion and scalar models (summarized in sect. 4), we conjecture that the necessary and sufficient condition for $\delta \Pi \geqslant 0$ for all values of the couplings is that the mass matrix responsible for the splitting transform as the third component of an isovector. The standard model with arbitrary mixings among $N$ generations of quark and/or lepton doublets falls into this category. However, it is possible to construct models for which the mass matrix transforms otherwise, and we give an example of both a scalar and a fermion model for which one can have $\delta \Pi<0$.

An outline of this paper is as follows: in sect. 2 we show how the deviation of $\rho$ from $l$ is determined by the breaking of the global isospin symmetry, distinguishing carefully between different types of breaking, for both fermions and bosons transforming according to arbitrary representations of the gauge group. In sect. 3 we derive, both for scalars and fermions, a new formula for $\delta \Pi$. We apply the formula immediately to prove $\delta \Pi(0) \geqslant 0$ in a couple of special cases. In sect. 4, we take up several special models; first, we give simple examples of scalar and fermion multiplets which can yield either sign for $\delta \Pi$. Then we consider other special cases of our general conjecture. Finally, sect. 5 concludes with some remarks concerning related matters.

\section{The standard model, isospin, and heavy particles}

The lagrangian of the standard $S U(2) \otimes U(1)$ electroweak model (without fermions for now) can be written as [12]

$$
\begin{aligned}
\mathcal{L}= & -\frac{1}{2} \operatorname{Tr}\left(w_{\mu \nu}^{\dagger} w^{\mu \nu}\right)-\frac{1}{4} B_{\mu \nu} B^{\mu \nu} \\
& -\frac{1}{2} \operatorname{Tr}\left(D^{\mu} \Phi\right)^{\dagger}\left(D_{\mu} \Phi\right)-V\left(\Phi^{\dagger} \Phi\right) .
\end{aligned}
$$

\footnotetext{
* For application of this symmetry to a gencral classification of heavy Higgs effects. sce ref. [12].
} 
The (matrix) fields appearing here are defined as follows: Let $B_{\mu}$ be the U(1) singlet and $w^{\mu} \equiv-\frac{1}{2} i w_{a}^{\mu} \sigma_{a}$ be the $\mathrm{SU}(2)$ triplet gauge potentials. Then the field strengths are

$$
\begin{aligned}
& B_{\mu \nu}=\partial_{\mu} B_{\nu}-\partial_{\nu} B_{\mu}, \\
& w_{\mu \nu}=\partial_{\mu} w_{\nu}-\partial_{\nu} w_{\mu}+g\left[w_{\mu}, w_{\nu}\right] .
\end{aligned}
$$

The usual Higgs scalar doublet is

$$
\phi=\left(\begin{array}{c}
\phi_{+} \\
\phi_{0}
\end{array}\right)
$$

and the conjugate doublet is

$$
\tilde{\phi}=i \sigma_{2} \phi^{*}=\left(\begin{array}{c}
\phi_{0}^{\dagger} \\
-\phi_{-}
\end{array}\right) .
$$

Together they compose the columns of the matrix

$$
\Phi=\left(\begin{array}{ll}
\tilde{\phi} & \phi
\end{array}\right) .
$$

The Higgs potential $V$ is

$$
V=\frac{1}{2} \lambda\left(\frac{1}{2} \operatorname{Tr}\left(\Phi^{\dagger} \Phi\right)-\sigma^{2}\right)^{2} .
$$

The covariant derivative is defined as

$$
D_{\mu} \Phi=\partial_{\mu} \Phi+g w_{\mu} \Phi-\frac{1}{2} i g^{\prime} B_{\mu} \Phi \sigma_{3} .
$$

In the absence of the hypercharge coupling $\left(g^{\prime}=0\right)$, the lagrangian possesses a global $\mathrm{SU}(2) \otimes \mathrm{SU}(2)^{\prime}$ invariance $[1,12]$ given by

$$
\begin{aligned}
& \Phi \rightarrow G \Phi H^{\dagger}, \\
& w_{\mu} \rightarrow G w_{\mu} G^{\dagger}, \\
& B_{\mu} \rightarrow B_{\mu},
\end{aligned}
$$

where $G, H$ are (independent) $\mathrm{SU}(2)$ matrices.

The presence of $\sigma_{3}$ in the hypercharge interaction explicitly breaks the additional global SU(2)' symmetry associated with $H$. It is worth noting that radiative corrections to $\rho$ due to hypercharge do not occur at the one-loop level, so for one-loop calculations, the hypercharge coupling $g^{\prime}$ can indeed be neglected. Thus the additional $\mathrm{SU}(2)^{\prime}$ symmetry has interesting implications at the one-loop level. 
Because of spontaneous symmetry breakdown (SSB), $\Phi$ develops a classical field which, in the conventional unitary gauge, is represented as

$$
\langle\Phi\rangle=\sigma\left(\begin{array}{ll}
1 & 0 \\
0 & 1
\end{array}\right)
$$

(The magnitude $\sigma$ is, of course, gauge invariant and we'll choose energy units so that $\sigma \equiv 1$ henceforth.) Although SSB breaks the $\mathrm{SU}(2) \otimes \mathrm{SU}(2)^{\prime}$ global symmetry, there remains a "diagonal" unbroken $\mathrm{SU}(2)$,

$$
\begin{gathered}
\Phi \rightarrow G \Phi G^{\dagger}, \\
w_{\mu} \rightarrow G w_{\mu} G^{\dot{\dagger}},
\end{gathered}
$$

which we shall call isospin invariance. Thus, under isospin, the Higgs field is the sum of an isoscalar $(\operatorname{Tr} \Phi)$ and an isovector $\left(\Phi-\frac{1}{2}(\operatorname{Tr} \Phi) 1\right)$. The vector boson $w_{\mu}$, being traceless, remains an isovector, just as it was under weak isospin.

Another, independent source of isospin breaking comes when fermions are included with Yukawa interactions. For example, in the standard model, with a left-handed quark doublet $\psi_{\mathrm{L}}=\left(\begin{array}{c}U_{\mathrm{I}} \\ D_{\mathrm{L}}\end{array}\right)$ and right-handed singlets $U_{\mathrm{R}}, D_{\mathrm{R}}$, gaugeinvariant terms occur of the form

$$
y_{1} \bar{\psi}_{\mathrm{L}} \tilde{\phi} U_{\mathrm{R}}+y_{2} \bar{\psi}_{\mathrm{L}} \phi D_{\mathrm{R}}
$$

which may be written alternatively as

$$
y \bar{\psi}_{\mathrm{L}} \Phi \psi_{\mathrm{R}}+\Delta y \bar{\psi}_{\mathrm{L}} \Phi \sigma_{3} \psi_{\mathrm{R}}
$$

where we defined

$$
\psi_{\mathrm{R}} \equiv\left(\begin{array}{c}
U_{\mathrm{R}} \\
D_{\mathrm{R}}
\end{array}\right)
$$

The first term $(y)$ possesses the additional $\mathrm{SU}(2)^{\prime}$ invariance

$$
\psi_{\mathrm{R}} \rightarrow H \psi_{\mathrm{R}}, \quad \Phi \rightarrow \Phi H^{\dagger}
$$

but this symmetry is broken by the second term $(\Delta y)$. (We should remark that because of hypercharge, it is not possible to renormalize the theory with $\Delta y=0$, so this term must naturally be included.) Thus, except for the hypercharge corrections and the mass splitting within isospin multiplets, the full theory is isospin invariant:

$$
\begin{array}{rlrl}
\Phi & \rightarrow G \Phi G^{\dagger}, & w_{\mu} \rightarrow G w_{\mu} G^{\dagger}, \\
\psi_{\mathrm{L}} \rightarrow G \psi_{\mathrm{L}}, & \psi_{\mathrm{R}} \rightarrow G \psi_{\mathrm{R}} .
\end{array}
$$


In the subsequent sections, we shall focus on the one-loop radiative corrections to $\rho$ from fermions and from scalar bosons, in arbitrary isospin multiplets. For this purpose, it is useful to discuss the symmetry properties in general.

The basis for a general representation of SU(2) may be characterized as a tensor field $\psi_{t_{1}, t_{2}} \ldots i_{n}$ with indices taking values, say, $\ldots 1$ transforming as

$$
\psi_{i_{1} \ldots i_{n}} \rightarrow G_{i_{1},} \cdots G_{i_{n} / n} \psi_{J_{1}} \cdots i_{n} .
$$

An irreducible representation (of spin $\frac{1}{2} n$ ) is characterized by a field with $n$ indices, totally symmetric in its indices. Of course, the hermitian conjugate field $\psi_{1_{1}}^{+} \ldots$, n $_{n}$ transforms according to the complex conjugate representation, $\psi^{i_{1}}{ }^{-} i_{n}$ but indices may be lowered using the invariant "metric" tensor $\varepsilon_{i j}=-\varepsilon_{j i}$. Thus it is unnecessary, although sometimes convenient, to deal with mixed tensors.

For fermions, the most general gauge-invariant mass terms are of the form

$$
m \bar{\psi}_{\mathrm{L}_{1}, \ldots j_{n}} \psi_{\mathrm{R}_{1} \ldots j_{n}}+\text { h.c. }
$$

where we have distinguished between left (L) and right (R) chiral fields $\psi_{L}$ and $\psi_{R}$. One may have gauge-invariant Yukawa couplings to the Higgs field contributing to the mass after SSB:

$$
y_{2} \bar{\psi}_{L_{j_{1}} \cdots j_{n}} \phi_{j_{1}} \chi_{\mathrm{R} j_{2} \cdots j_{n}}+y_{1} \bar{\psi}_{L_{j_{1}} \ldots j_{n}} \tilde{\phi}_{j_{1}} \xi_{\mathrm{R}_{2} \ldots j_{n}}+\text { h.c. }
$$

where we assume the fields $\chi$ and $\xi$ carry appropriate hypercharge so that the terms are $\mathrm{U}(1)$ invariant. Without loss of generality, we may assume these fields transform as irreducible representations of SU(2). As with the standard doublets and singlets, it is convenient to rewrite these terms as follows:

Let $\Phi_{j}^{k}$ be defined as before, i.e.

$$
\Phi_{j}^{-}=\tilde{\phi}_{j}, \quad \Phi_{j}^{-}=\phi_{j},
$$

and define a fermion multiplet $X_{k, j_{2}} \ldots j_{n}$ according to

$$
X_{-, j_{2} \cdots j_{n}} \equiv \xi_{j_{2} \cdots j_{n}}, \quad X_{-, j_{2} \cdots j_{n}} \equiv \chi_{j_{2} \cdots j_{n}} .
$$

(For notational simplicity, the chiral subscript $R$ has been suppressed.) In this notation, the Yukawa terms are

$$
y \bar{\psi}_{j_{1} \ldots j_{n}} \Phi_{j_{1}}^{k} X_{k, j_{2} \ldots j_{n}}+\Delta y \bar{\psi}_{j_{1} \ldots j_{n}}\left(\Phi \sigma_{3}\right)_{j_{1}}^{k} X_{k, j_{2} \ldots j_{n}}+\text { h.c. }
$$


As before, we may define a new $\mathrm{SU}(2)^{\prime}$ transformation under which $\psi_{\mathrm{L}}$ is a singlet but $\Phi$ and $X$ are doublets:

$$
\begin{aligned}
\Phi_{j}^{k} & \rightarrow \Phi_{j}^{k^{\prime}}\left(H^{\dagger}\right)_{k^{\prime} k}, \\
X_{k, j_{2} \cdots j_{n}} & \rightarrow H_{k k^{\prime}}, X_{k^{\prime}, j_{2} \cdots y_{n}} .
\end{aligned}
$$

Under this new $\operatorname{SU}(2)^{\prime}$, the first term $(y)$ is a singlet, while the second $(\Delta y)$ is an isovector. (Of course, one could equally well choose $\left(\sigma_{3}\right)_{k}^{k^{\prime}} X_{k^{\prime}, j_{2}} \ldots$, $_{n}$ to be the doublet under this $\mathrm{SU}(2)^{\prime}$, an observation which will be useful later. Had we chosen $\sigma_{3} X$ as our doublet, then the transformation properties of the two Yukawa terms would be reversed.) One can easily check that, neglecting hypercharge interactions $\left(g^{\prime}=0\right)$, the contributions to the lagrangian involving the covariant derivatives are also invariant under this new $\mathrm{SU}(2)^{\prime}$.

After SSB, $\left\langle\Phi_{j}^{k}\right\rangle=\sigma \delta_{j}^{k}$ so the theory in lowest order remains invariant under isospin, defined as the "diagonal" $\mathrm{SU}(2)$ given by requiring $H=G$. Thus, quite generally, the first Yukawa term $(y)$ is isospin invariant while the second $(\Delta y)$ transforms as the third component of an isovector.

This discussion generalizes to allow the addition of other fields which mix with $\psi_{L}, \chi_{R}$, and $\xi_{R}$, as well as the interchange of left and right or the inclusion of Majorana fields. Generally, the mass matrix will transform as an isosinglet plus an isovector but, unlike the preceding example, with several multiplets it may not be possible to define an isospin so that the breaking transforms as the third component of an isovector. This is best illustrated by an example, which will be useful later. Suppose we have a model consisting of two weak left doublets with different hypercharge

$$
L_{a}=\left(\begin{array}{c}
E^{+} \\
N
\end{array}\right), \quad L_{b}=\left(\begin{array}{c}
\nu \\
e^{-}
\end{array}\right)
$$

and four right weak singlets $R^{+}, R_{a}^{0}, R_{b}^{0}, R^{-}$. The usual sort of Yukawa interactions are

$$
\bar{L}_{a} \Phi\left(y_{a}+\Delta y_{a} \sigma_{3}\right) R_{a}+\bar{L}_{b} \Phi\left(y_{b}+\Delta y_{b} \sigma_{3}\right) R_{b}+\text { h.c. }
$$

where we define right doublets under global $\mathrm{SU}(2)^{\prime}$ to be

$$
R_{a}=\left(\begin{array}{c}
R^{+} \\
R_{a}^{0}
\end{array}\right), \quad R_{b}=\left(\begin{array}{c}
R_{b}^{0} \\
R^{-}
\end{array}\right)
$$

However, since $R_{a}^{0}$ and $R_{b}^{0}$ have the same hypercharge, then the possibility exists of having additional Yukawa terms of the form

$$
h \bar{L}_{a} \phi R_{b}^{0}+h^{\prime} \bar{L}_{b} \tilde{\phi} R_{a}^{0}=\frac{1}{2} h \bar{L}_{a} \Phi \sigma_{-} R_{b}+\frac{1}{2} h^{\prime} \bar{L}_{b} \Phi \sigma_{+} R_{a}
$$


The general form of the mass matrix is

$$
\left(\bar{E}-\bar{N} \bar{\nu} \bar{e}^{-}\right)\left(\begin{array}{cccc}
g_{a} & 0 & 0 & 0 \\
0 & g_{a}^{\prime} & h & 0 \\
0 & h^{\prime} & g_{h}^{\prime} & 0 \\
0 & 0 & 0 & g_{h}
\end{array}\right)\left(\begin{array}{c}
R^{+} \\
R_{a}^{0} \\
R_{b}^{0} \\
R
\end{array}\right),
$$

where $g_{a}=y_{a}+\Delta y_{a}, g_{a}^{\prime}=y_{a}-\Delta y_{a}$, and similarly for $g_{b}, g_{b}^{\prime}$. In general, there is no redefinition of isospin possible for which the mass terms commute with the third component of isospin. (Such a redefinition is, in fact, possible when $h g_{b}^{\prime}=-h^{\prime} g_{a}^{\prime}$.) Therefore, one generally has terms in the mass matrix breaking isospin in directions other than the third.

The present model differs from "generational" types of models, where different multiplets of the same weak isospin also have the same hypercharge. Such models continue to admit a definition of isospin for which the third component remains conserved. Although mixing of the type we have described can be excluded by discrete symmetries, in general we know of no reason not to expect such a phenomenon so that no component of isospin remains conserved.

Scalar fields are actually more complicated from this point of view, because, in addition to trilinear terms of the type discussed above for fermions, there are quartic terms which, after SSB, contribute to the boson mass-squared matrix. Henceforth, we'll simply call this the boson mass matrix for short. For simplicity, we will first illustrate the complications for the case of another scalar doublet $\chi$ which, we will suppose, does not develop a classical expectation value. For now, let us suppose the field carries a non-zero additive quantum number different from $\phi($ or $\tilde{\phi})$ [either hypercharge or an independent $U(1)]$ so that it always occurs as $\chi_{i}^{\dagger} \chi$. Then we may couple it to the Higgs scalar in the form

$$
\alpha \chi^{\dagger} \chi \phi^{\dagger} \phi+\beta \chi^{\dagger} \boldsymbol{\sigma} \chi \cdot \phi^{\dagger} \boldsymbol{\sigma} \phi .
$$

To discuss the isospin properties after SSB, we need to introduce

$$
X=(\tilde{\chi} \chi),
$$

analogous to $\Phi$. Then we may write the previous coupling as

$$
\frac{1}{2} \alpha \operatorname{Tr}\left(X^{\dagger} X\right) \operatorname{Tr}\left(\Phi^{\dagger} \Phi\right)+\frac{1}{4} \beta \operatorname{Tr}\left(X^{\dagger} \boldsymbol{\sigma} X \sigma_{3}\right) \cdot \operatorname{Tr}\left(\Phi^{\dagger} \boldsymbol{\sigma} \Phi \sigma_{3}\right) .
$$

The first term is invariant under

$$
\Phi \rightarrow G \Phi H_{1}^{\dagger}, \quad X \rightarrow G \Phi H_{2}^{\dagger},
$$

Note that the SU(2) matrices on the right, $H_{1}$ and $H_{2}$, may be different for $\Phi$ and $\chi$. (The ones on the left must be the same to preserve the invariance of the kinetic 
energy, involving the vector field which transforms as $w_{\mu} \rightarrow G w_{\mu} G^{\dagger}$.) The second term above breaks this $\mathrm{SU}(2) \otimes \mathrm{SU}(2)^{\prime} \otimes \mathrm{SU}(2)^{\prime \prime}$ symmetry. After $\mathrm{SSB},\langle\Phi\rangle=\sigma \mathrm{l}$, and we must choose $H_{1}=G$, but we are still free to specify $H_{2}$ as we please, i.e., except for the hypercharge coupling and the second term $(\beta)$ above, the theory remains invariant under $\mathrm{SU}(2)^{\prime} \otimes \mathrm{SU}(2)^{\prime \prime}$. This contribution to the mass matrix becomes, after SSB,

$$
\frac{1}{2} \operatorname{Tr}\left(X^{\dagger} X\right)+\frac{1}{2} \beta \operatorname{Tr}\left(X^{\dagger} \sigma_{3} X \sigma_{3}\right)
$$

It is most convenient to define isospin for $X$ as $X \rightarrow G X$ (i.e. $H_{2}=1$ ), since then the symmetry-breaking term $(\beta)$ transforms as the third component of an isovector ${ }^{\star}$. If $\chi$ does not carry an independent $U(1)$, and has hypercharge equal to $\phi$, then another gauge-invariant quartic term contributing to the mass matrix is

$$
\frac{1}{2} \gamma \chi^{\dagger} \boldsymbol{\sigma} \tilde{\chi} \cdot \tilde{\phi}^{\dagger} \boldsymbol{\sigma} \phi+\text { h.c. }
$$

which may be conveniently written as

$$
\frac{1}{4} \gamma\left[\operatorname{Tr}\left(X^{\dagger} \sigma_{a} X \sigma_{b}\right) \operatorname{Tr}\left(\Phi^{\dagger} \sigma_{a} \Phi \sigma_{b}\right)-\operatorname{Tr}\left(X^{\dagger} \sigma_{a} X \sigma_{3}\right) \operatorname{Tr}\left(\Phi^{\dagger} \sigma_{a} \Phi \sigma_{3}\right)\right] .
$$

The first term in the brackets is invariant under

$$
\Phi \rightarrow G \Phi H^{\dagger}, \quad X \rightarrow G X H^{\dagger}
$$

(Note that in this case, it is most convenient to choose $X$ to transform like $\Phi$.) The second term breaks this $\mathrm{SU}(2) \otimes \mathrm{SU}(2)^{\prime}$ down to the original weak $\operatorname{SU}(2)(H=1)$. Under the $\operatorname{SU}(2)^{\prime}(G=1, H$ arbitrary) it transforms as the 3,3 component of a second-rank tensor.

After SSB, this contribution to the mass is

$$
\frac{1}{2} \gamma\left[\operatorname{Tr}\left(X^{\dagger} \sigma_{a} X \sigma_{\omega}\right)-\operatorname{Tr}\left(X^{\dagger} \sigma_{3} X \sigma_{3}\right)\right]
$$

Thus, the first term remains invariant under isospin (defined as $G=H$ ), while the second transforms like a second-rank tensor. Suppose instead we had chosen to define isospin for $X$ as $X \rightarrow G X$. Then the symmetry breaking of the mass term is isovector, but it no longer commutes with the third component of isospin.

So another way to state the conjectured theorem is as follows: The one-loop radiative corrections to $\delta \rho$ due to mass splittings are always positive if there exists a definition of isospin such that the symmetry breaking of the mass matrix transforms as the third component of an isovector. (By mass matrix, we mean, after SSB. the mass terms for fermions and the mass-squared terms for bosons.)

* If, on the contrary, we chose $H_{2}=G$ in our definition of isospin so that $X$ transforms as $\Phi$, then the breaking would be classified as a tensor. 
The two terms discussed above can be combined in the form

$$
\frac{1}{4} \gamma \operatorname{Tr}\left(X^{\dagger} \sigma_{a} X \sigma_{b}\right) \operatorname{Tr}\left(\Phi^{\dagger} \sigma_{a} \Phi \sigma_{b}\right)+\frac{1}{4}(\beta-\gamma) \operatorname{Tr}\left(X^{\dagger} \sigma_{a} X \sigma_{3}\right) \operatorname{Tr}\left(\Phi^{\dagger} \sigma_{a} \Phi \sigma_{3}\right) .
$$

We may summarize our previous discussion as follows: After SSB, the symmetry structure may be described as isovector or isotensor depending on the definition of isospin chosen. However, there are two special cases worthy of note: (i) $\gamma=0$, for which there is a definition of isospin so that the symmetry breaking transforms as the third component of an isovector, and (ii) $\beta=\gamma$, for which there is a definition under which isospin symmetry remains unbroken and $\delta \rho=0$ to one loop order.

A third alternative model occurs when $\chi$ is a hypercharge singlet, in which case the preceding term $(\gamma)$ is not allowed but instead we may have two other terms:

$$
\delta \phi^{\dagger} \tilde{\chi} \phi^{\dagger} \phi+\frac{1}{2} \varepsilon \chi^{\dagger} \sigma \tilde{\chi} \cdot \phi^{\dagger} \boldsymbol{\sigma} \phi+\text { h.c. }
$$

which may be written as

$$
{ }_{2}^{1} \delta \operatorname{Tr}\left(X^{\dagger} X \sigma_{1}\right) \operatorname{Tr}\left(\Phi^{\dagger} \Phi\right)-\frac{1}{2} \varepsilon \operatorname{Tr}\left(X^{\dagger} \boldsymbol{\sigma} X \sigma_{1}\right) \operatorname{Tr}\left(\Phi^{\dagger} \boldsymbol{\sigma} \Phi \sigma_{3}\right) .
$$

One may also add terms of the $\alpha$ and $\beta$ type as before. As in the first model (with only $\alpha$ and $\beta$ terms), it is most convenient to define isospin asymmetrically as

$$
\Phi \rightarrow G \Phi G^{\dagger}, \quad X \rightarrow G X .
$$

With this definition, the $\alpha$ and $\delta$ terms are invariant but the $\beta$ and $\varepsilon$ terms both transform as the third components of isovectors.

This exhausts the discussion of an additional scalar boson doublet. The generalization to arbitrary weak isospin representations follows a similar path as for fermions. except that now, in addition to possible trilinear couplings, we may have an abundance of quartic terms which, after SSB, contribute to the mass matrix.

It is useful to standardize the notation for these symmetry operations. We shall denote the generators of weak isospin as $T_{a}$ and the generators of isospin $U_{a}$ (regardless of which definition is adopted.) The difference $S_{a}=U_{a}-T_{a}$ generates the additional SU(2)' transformation. Clearly, $\left[T_{a}, S_{b}\right]=0$, so $T_{a}, S_{a}$, and $U_{a}$ have the commutation relations of orbital, spin, and total angular momenta.

In this language, our conjecture is as follows: Let $\mathcal{N}(x)$ denote the quadratic terms in the lagrangian density due to matter fields ${ }^{\star}$. Suppose there is a definition of isospin such that $\left[U_{3}, \Im \pi(x)\right]=0$. Then the one-loop radiative corrections to $\delta \Pi$ due

* By matter fields, we mean all fermions and those scalar fields other than the Higgs scalar. : $(x)$ denotes their mass for fermions and their mass-squared for scalars. It would be interesting to extend the conjecture to the contributions of vector bosons other than the $W$ and $Z_{0}$, but we have not investigated this case. 
to these matter fields are non-negative. Moreover, they vanish only if isospin is conserved $\left[U_{a}, \mathfrak{T}(x)\right]=0$.

\section{General formalism and elementary applications}

The polarization tensor for the vector boson self-energy is formally given as

$$
\Pi_{a b}^{\mu \nu}(q)=-i \int \mathrm{d}^{4} x \mathrm{e}^{i q \cdot x} \mathrm{~T}\left\langle 0\left|J_{a}^{\mu}(x) J_{b}^{\nu}(0)\right| 0\right\rangle+\cdots,
$$

where the ellipses represent possible seagull graphs (for scalars), renormalization counterterms, and terms necessary to render the time-ordered product Lorentz covariant. As a consequence of minimal coupling the source $J_{a}^{\mu}(x)$ of the vector boson field is, for those "matter" fields other than the Higgs field and the vector boson field itself, identical with their contribution to the weak isovector current. Of course, the longitudinal form factor $\Pi_{a b}\left(q^{2}\right)$ vanishes in the symmetric theory; that is, $\Pi_{a b}(0)$ is non-zero only because of spontaneous symmetry breakdown. We have found it useful to express this directly by noting that

$$
q_{\mu} q_{\nu} \Pi_{a b}^{\mu \nu}=q^{2} \Pi_{a b}\left(q^{2}\right)=-i \int \mathrm{d}^{4} x \mathrm{e}^{i q \cdot x} \mathrm{~T}\left\langle 0\left|\partial \cdot J_{a}(x) \partial \cdot J_{b}(0)\right| 0\right\rangle+\cdots .
$$

Here the ellipses represent possible counterterms and equal-time terms. (Seagulls are cancelled by Schwinger terms in the usual way $[13,14]$.) It will be important later that the only possible equal-time term is a constant, independent of $q^{2}$, as one can easily show. Now what is the divergence $\partial \cdot J_{a}(x)$ of the contribution of "matter" fields to the weak current? By a well-known theorem $[13,14]$ it is just the change $\delta E_{a}(x)$ in the lagrangian due to an infinitesimal, global weak $S U(2)$ transformation on the matter fields, keeping the Higgs field $\phi$ and vector boson field $W_{a}^{\mu}$ fixed. By expanding the exponential, we may then obtain

$$
\Pi_{a b}(0)=\frac{1}{8} i \int \mathrm{d}^{4} x x^{2} \mathrm{~T}\left\langle 0\left|\delta \mathscr{L}_{a}(x) \delta E_{b}(0)\right| 0\right\rangle+\cdots .
$$

The ellipses represent only possible renormalization counterterms. No equal-time terms contribute. As a practical matter, it is frequently easier to calculate $\delta E_{a}$ as follows: If we consider just those terms in the lagrangian coming from the addition of matter fields, then these are SU(2) gauge invariant by themselves. Therefore, instead of rotating the matter fields, we can calculate $\delta e_{a}$ as minus the change in these terms obtained by performing a global weak SU(2) transformation on the Higgs $\phi$ and vector fields $W_{a}^{\mu}$ alone, keeping the matter fields fixed. This means the 
change can be calculated by the replacements

$$
\begin{gathered}
\phi \rightarrow i \frac{1}{2} \sigma_{a} \phi, \\
W_{b}^{\mu} \rightarrow \varepsilon_{a b c} W_{c}^{\mu} .
\end{gathered}
$$

These developments lead to an extremely simple formula for the one-loop corrections to $\Pi_{a b}$, but we shall need some additional notation in order to treat the general case. First we shall treat scalar fields and later deal with the spin complications associated with fermions. Let $\chi_{m}$ denote all the scalar "matter" fields and let $M^{2}$ denote the mass-squared matrix after spontaneous breakdown (i.e. $\left.\chi_{n}^{\dagger}\left(M^{2}\right)_{n m} \chi_{m}\right)$ represents the terms quadratic in the scalar fields. Let $T_{a}$ represent the weak charge matrices so that $\delta \mathfrak{E}_{a}=i\left[T_{a}, M^{2}\right]$. Then the one-loop contribution may be represented as

$$
q^{2} \Pi_{a b}\left(q^{2}\right)=-i \int \frac{\mathrm{d}^{n} k}{(2 \pi)^{n}} \operatorname{Tr}\left[\delta \varrho_{a} \Delta\left(k_{+}\right) \delta \varrho_{b} \Delta\left(k_{-}\right)\right]
$$

where we have denoted the scalar propagator as $\Delta(k) \equiv\left[k^{2}-M^{2}\right]^{-1}$ and the trace denotes the sum over all scalar matter fields. The loop momenta $k_{+}$and $k_{-}$can be parametrized in a general way as $k_{ \pm}=k+\left(\xi \pm \frac{1}{2}\right) q$, where $\xi$ is an arbitrary parameter. To obtain $\Pi_{a b}(0)$, we must expand to second order in $q$. Since the integral must be independent of the choice of $\xi$, this leads to a number of alternative forms for the result (see appendix $\mathrm{A}$ ), but we find the following most symmetric:

$$
\Pi_{a b}(0)=\frac{1}{8} i \int \frac{\mathrm{d}^{n} k}{(2 \pi)^{n}} \operatorname{Tr}\left[\delta E_{a} \frac{\partial \Delta}{\partial k_{\mu}} \delta \varrho_{b} \frac{\partial \Delta}{\partial k_{\mu}}\right] .
$$

Using

$$
\frac{\partial \Delta}{\partial k_{\mu}}=-\Delta \frac{\partial \Delta^{-1}}{\partial k_{\mu}} \Delta=-2 k_{\mu} \Delta^{2}
$$

this can also be written as

$$
\Pi_{a b}(0)=\frac{1}{2} i \int \frac{\mathrm{d}^{4} k}{(2 \pi)^{4}} k^{2} \operatorname{Tr}\left[\delta \mathcal{L}_{a} \Delta^{2} \delta \varrho_{b} \Delta^{2}\right]
$$

Note that this converges in four dimensions. Now

$$
\Delta \delta \mathscr{L}_{\alpha} \Delta=i \Delta\left[T_{a}, \Delta^{-1}\right] \Delta=-i\left[T_{a}, \Delta\right]
$$

so the result is simply

$$
\Pi_{a b}(0)=-\frac{1}{2} \int \frac{\mathrm{d}^{4} k}{(2 \pi)^{4}} k^{2} \operatorname{Tr}\left(\left[T_{a}, \Delta\right]\left[T_{b}, \Delta\right]\right),
$$


where we have Wick-rotated to the euclidean form of the integral. No counterterms, or equal-time terms need be added; this is the full answer. The diagonal elements $\Pi_{a a}$ are manifestly positive, since $i\left[T_{a}, \Delta\right]$ is a hermitian matrix. (This could also be established via a dispersion relation but it is nice to see it here.) The result may also be written as

$$
\Pi_{a b}(0)=\frac{1}{2} \int \frac{\mathrm{d}^{4} k}{(2 \pi)^{4}} k^{2} \operatorname{Tr}\left(\Delta\left[T_{a},\left[T_{b}, \Delta\right]\right]\right),
$$

which bears a resemblance to Dashen's current algebra formula for Goldstone boson masses [15].

As a simple application of this formula, consider a model of arbitrary scalar fields for which the mass matrix commutes with $T_{3}$. (This occurs for example for quartic couplings of the type $\phi^{+} \sigma_{a} \phi \chi_{j_{1}}^{\dagger} \ldots j_{n}\left(\sigma_{a}\right)_{j_{1}}^{k_{1}} \chi_{k_{\nu_{2}} \ldots j_{n}}$.) Then clearly $\Pi_{33}(0)=0$, so that

$$
\delta \mathrm{II}=\mathrm{II}+-\geqslant 0
$$

Thus any such model can only increase $\rho$.

Fermions present some complications which can be conveniently organized as follows: Models are generally presented in terms of chiral fields which we arrange as (column) vectors left $L_{m}$ and right $R_{n}$. After SSB, the mass terms assume the general form $\bar{L}_{m}(M)_{m n} R_{n}+$ h.c. $\equiv \bar{L} M R+$ h.c. (The matrix $M$ is not necessarily square.) Because the massive fermions are conveniently represented as Dirac fields, we take these chiral fields in four-component form. We then gather the left and right fields together in a single column vector so the complete mass matrix takes the block form

$$
\Re=\left(\begin{array}{cc}
0 & M \\
M^{+} & 0
\end{array}\right)
$$

Similarly, we define weak charges $T_{a \mathrm{~L}}$ and $T_{a \mathrm{R}}$ for the left and right chiral fields, respectively. They can be grouped together as

$$
T_{a}=\left(\begin{array}{cc}
T_{a \mathrm{~L}} & 0 \\
0 & T_{a \mathrm{R}}
\end{array}\right)
$$

so that the divergence is represented as

$$
\delta \mathfrak{L}_{a}=i\left[T_{a}, \mathfrak{\Re}\right]=i\left(\begin{array}{cc}
0 & \Lambda_{a} \\
-\Lambda_{a}^{\dagger} & 0
\end{array}\right)
$$

where $\Lambda_{a}=T_{a \mathrm{~L}} M-M T_{a \mathrm{R}}$. The Feynman propagator is $S(k)=(\not k-\mathscr{N})^{-1}=$ 
$\Delta(k+\mathcal{N})$, with

$$
\Delta(k)=\left(k^{2}-\mathfrak{M}^{2}\right)^{-1}=\left(\begin{array}{cc}
\Delta_{\mathrm{L}} & 0 \\
0 & \Delta_{\mathrm{R}}
\end{array}\right) \text {. }
$$

Here we have

$$
\mathbb{M}^{2}=\left(\begin{array}{cc}
M M^{\dagger} & 0 \\
0 & M^{\dagger} M
\end{array}\right)
$$

and $\Delta_{\mathrm{L}}{ }^{1} \equiv k^{2}-M M^{\dagger}$ and $\Delta_{\mathrm{R}}{ }^{1}=k^{2}-M^{\dagger} M$. Note that $\Delta \mathcal{O R}=$ oR $\Delta$. Finally, we need to introduce the projectors $P_{\mathrm{L}}=\frac{1}{2}\left(1-\gamma_{5}\right)$ and $P_{\mathrm{R}}=\frac{1}{2}\left(1+\gamma_{5}\right)$, and

$$
P=\left(\begin{array}{cc}
P_{\mathrm{l}} & 0 \\
0 & P_{\mathrm{R}}
\end{array}\right)
$$

Then the one-loop contribution of all fermions may be written succinctly as

$$
q^{2} \Pi_{a b}\left(q^{2}\right)=-i \int \frac{\mathrm{d}^{n} k}{(2 \pi)^{n}} \operatorname{Tr}\left[P \delta E_{a} S\left(k_{-}\right) P \delta E_{b} S(k \ldots)\right]+\cdots
$$

where the trace includes a sum over Dirac indices. Now, it is convenient to eliminate the $\gamma_{s}$ dependence at once, noting that $\left[P, \delta \mathcal{L}_{a} S(k)\right]=0$, and $P^{2}=P$, and the $\gamma_{s}$ term gives no contribution to the Dirac trace. Proceeding as before, one can derive alternative forms for $\Pi_{a b}(0)$, including

$$
\mathrm{II}_{a b}(0)=-\frac{1}{8} i \int \frac{\mathrm{d}^{n} k}{(2 \pi)^{n}} \operatorname{Tr}\left[\delta \mathrm{E}_{a} \frac{\partial S}{\partial k_{\mu}} \delta E_{b} \frac{\partial S}{\partial k_{\mu}}\right]+\cdots
$$

Noting

$$
\frac{\partial S}{\partial k_{\mu}}=-S \frac{\partial S^{-1}}{\partial k_{\mu}} S=-S \gamma_{\mu} S
$$

and

$$
S \delta \mathcal{L}_{a} S=i S\left[T_{a}, M\right] S=-i\left[T_{a}, S\right]
$$

we find

$$
\Pi_{a b}(0)=\frac{1}{8} i \int \frac{\mathrm{d}^{n} k}{(2 \pi)^{n}} \operatorname{Tr}\left(\left[T_{a}, S(k)\right] \gamma_{\mu}\left[T_{b}, S(k)\right] \gamma_{\mu}\right)+\cdots
$$

Unlike the boson case, the integral does not converge in four dimensions, and a 
counter-term is required. However, we shall soon see that the (logarithmic) divergence is proportional to $\delta_{a b}$ (an isosinglet), so when we form the difference to calculate $\delta \rho$, the divergences will cancel. Thus, as expected, $\delta \rho$ is finite and calculable. At this point, it is convenient to carry out the Dirac trace which leads to

$$
\Pi_{a b}(0)=-\frac{1}{2} \int \frac{\mathrm{d}^{4} k}{(2 \pi)^{4}} \operatorname{Tr}\left(2\left[T_{a}, \Re \Delta\right]\left[T_{b}, 刃 \Re \Delta\right]+k^{2}\left[T_{a}, \Delta\right]\left[T_{b}, \Delta\right]\right)+\cdots .
$$

where we have again gone over to euclidean form. Alternatively, this may be written as

$$
\frac{1}{2} \int \frac{\mathrm{d}^{4} k}{(2 \pi)^{4}} \operatorname{Tr}\left(2 \mathfrak{N} \Delta\left[T_{a},\left[T_{b}, \Re \Delta\right]\right]+k^{2} \Delta\left[T_{a},\left[T_{b}, \Delta\right]\right]\right)+\cdots
$$

This latter form is particularly convenient for analyzing the divergence structure, which arises from the term of the form

$$
\operatorname{Tr}\left(\mathfrak{R} \Delta\left[T_{a},\left[T_{b}, \Re\right]\right] \Delta\right)
$$

However, because the symmetry breaking is via an isodoublet, one can show (see appendix B) that

$$
\left[T_{a},\left[T_{b}, \Re\right]\right]=\frac{1}{4} \delta_{a b} \Re
$$

Hence, the divergence is symmetric and will cancel in $\delta \rho$, as asserted earlier.

We may illustrate the formalism for a standard left doublet coupled to a pair of right singlets. With the notation introduced in the preceding section [eq. (2.11)], the relevant matrices are

$$
\mathfrak{R}=\left(\begin{array}{cc}
0 & y+\Delta y \sigma_{3} \\
y+\Delta y \sigma_{3} & 0
\end{array}\right), \quad T_{a}=\left(\begin{array}{cc}
\frac{1}{2} \sigma_{a} & 0 \\
0 & 0
\end{array}\right)
$$

The isospin generators are taken to be

$$
U_{a}=\left(\begin{array}{cc}
\frac{1}{2} \sigma_{a} & 0 \\
0 & \frac{1}{2} \sigma_{a}
\end{array}\right),
$$

so that $U_{a}$ commutes with $\mathscr{R}$. It can easily be seen that $\left[T_{3}, \Re^{2}\right]=0$, so that also $\left[T_{3}, \Delta\right]=0$. Moreover, $\left[T_{a},\left[T_{a}, \mathfrak{N} \Delta\right]\right]=\frac{1}{4} \Delta \mathscr{R}$ (not summed), independent of the 
choice of $a$. Hence the entire result for $\delta \Pi \equiv \Pi_{+-}-\Pi_{33}$ is

$$
\delta \mathrm{II}=-\frac{1}{4} \int \frac{\mathrm{d}^{4} k}{(2 \pi)^{4}} k^{2} \operatorname{Tr}\left(\left[T_{+}, \Delta\right][T, \Delta]\right) \geqslant 0 .
$$

To recover this result in more familiar form, write

$$
\Delta_{\mathrm{L}}=\Delta_{\mathrm{R}}=\left(\begin{array}{ll}
\left(k^{2}+m_{1}^{2}\right)^{-1} & \\
& \left(k^{2}+m_{2}^{2}\right)
\end{array}\right) \text {. }
$$

Then we find

$$
\delta \Pi=\frac{1}{4} f\left(m_{1}^{2}, m_{2}^{2}\right),
$$

where the ubiquitous function $f$ is defined by

$$
\begin{aligned}
f\left(m_{1}^{2}, m_{2}^{2}\right) & =\int \frac{\mathrm{d}^{4} k}{(2 \pi)^{4}} k^{2}\left(\frac{1}{k^{2}+m_{1}^{2}}-\frac{1}{k^{2}+m_{2}^{2}}\right)^{2} \\
& =\frac{1}{16 \pi^{2}}\left[m_{1}^{2}+m_{2}^{2}-\frac{2 m_{1}^{2} m_{2}^{2}}{m_{1}^{2}-m_{2}^{2}} \ln \frac{m_{1}^{2}}{m_{2}^{2}}\right] .
\end{aligned}
$$

From the integral representation, it can be seen that $f\left(m_{1}^{2}, m_{2}^{2}\right) \geqslant 0$ for all values of the masses. Moreover, the integrand is non-negative for all values of the internal loop momentum. Indeed, we have found in every case for which $\delta \Pi \geqslant 0$, the integrand is also non-negative for every value of the loop momentum. This is a great advantage of the momentum space representation (as opposed to Feynman parametric form or the explicit analytic dependence on the masses or coupling constants. This example also illustrates that those fermion models for which the first term in eq. (3.22) completely cancels in the difference $\delta \Pi$ have precisely the same form as the scalar case.

The preceding result may be easily generalized to any number of generations of left doublets mixing arbitrarily with right singlets. One may easily show that again $\left[T_{a},\left[T_{a}, M \Delta\right]\right]=\frac{1}{4} M \Delta$ (not summed on $a$ ), so that the first term in eq. (3.22) makes no contribution to $\delta \Pi$. The second term is not so simple, since the propagator $\Delta$ now involves mixing among many fields. However, it is clear that $\delta \Pi$ involves a coupling of the $T_{a}$ matrices up to total weak isospin $T=2$. So the product of the two propagators must also be coupled to $T=2$. But $\Delta$ contains at most $T=1$, because it involves the composition of doublets and singlets. Hence one may discard all terms 
in $\Delta$ other than the isovector pieces $\Delta^{(1)}$. Write

$$
\left[T_{3}, \Delta^{(1)}\right]=\left[U_{3}, \Delta^{(1)}\right]-\left[S_{3}, \Delta^{(1)}\right]
$$

We assumed that $\left[U_{3}, M\right]=0$, the first term gives zero. And all doublets are $S$-spin singlets in such models, so the second term is also zero. Hence $\left[T_{3}, \Delta^{(1)}\right]=0$ and

$$
\delta \Pi=-\frac{1}{4} \int \frac{\mathrm{d}^{4} k}{(2 \pi)^{4}} k^{2} \operatorname{Tr}\left(\left[T_{+}, \Delta^{(1)}\right]\left[T_{-}, \Delta^{(1)}\right]\right) \geqslant 0 .
$$

Consequently, additional generations with arbitrary mixing angles only add to $\delta \rho$. This result may obviously be generalized to allow the addition of left singlets as well, with additional gauge-invariant mass terms.

\section{Applications}

In this section we use the general formalism to calculate $\delta \rho$ for a number of specific cases for both scalar and fermion fields. These examples, and others which we do not present, are all consistent with our conjectured theorem.

Consider the case of a single scalar doublet $\chi$ possessing the same hypercharge as the Higgs field $\phi$. The transformation properties with respect to $U$ and $T$ spin of the resulting lagrangian were treated in some detail in sect. 2 . If we write

$$
\chi=\left(\begin{array}{c}
\pi^{+} \\
\sqrt{\frac{1}{2}}\left(\sigma+i \pi^{0}\right)
\end{array}\right)
$$

then the mass eigenstates in general are $\pi^{+}, \sigma$ and $\pi^{0}$. In general, the mass matrix depends on the parameters $\beta$ and $\gamma$ as well as a common, $T$-spin invariant mass term. Corresponding, the three mass eigenvalues, $m_{.+}, m_{0}$, and $m_{0}$ can be arranged arbitrarily. Using the formalism of sect. 3 , one finds that

$$
\delta \Pi=\int \frac{\mathrm{d}^{4} k}{(2 \pi)^{4}} k^{2}\left[\left(\Delta_{+}-\Delta_{0}\right)^{2}+\left(\Delta_{+}-\Delta_{0}\right)^{2}-\left(\Delta_{0}-\Delta_{\sigma}\right)^{2}\right],
$$

where $\Delta_{+} \equiv\left(k^{2}+m_{+}^{2}\right)^{-1}$, etc. The quantity in brackets in the integrand can be shown to be

$$
2\left(m_{+}^{2}-m_{0}^{2}\right)\left(m_{+}^{2}-m_{\sigma}^{2}\right) \Delta_{+}^{2} \Delta_{0} \Delta_{\sigma}
$$

Consequently, $\delta \Pi$ can have either sign. In particular, the integrand is negative definite when the charged particle mass lies between the two neutrals.

With regard to this model, two special cases were considered in sect. 2 . From the explicit form of the mass matrix one sees easily that the $U$-spin symmetric case $\beta=\gamma$ 
[see eq. (2.31)] corresponds to $m_{+}=m_{0}$ and consequent vanishing of $\delta \rho$. On the other hand, the limit $\gamma=0$ corresponds to $m_{0}=m_{\sigma}$, but $m_{+} \neq m_{0}$ due to isovector breaking in the 3 -direction, in which case $\delta \Pi \geqslant 0$. Thus this model illustrates all facets of our conjectured theorem.

We now consider a scalar model which is formally similar to the type of fermion models which we will come to presently. The model consists of a scalar multiplet $\chi$ of $T$-spin $J$ coupled to two $T$-spin $J-\frac{1}{2}$ multiplets $\xi_{1}, \xi_{2}$ by trilinear couplings with the Higgs field $\phi$. As was shown in sect. 2 this corresponds to isovector breaking in the third direction and so we expect $\delta \rho \geqslant 0$. Writing the field as a column vector

$$
\Phi=\left(\begin{array}{l}
x \\
\xi_{1} \\
\xi_{2}
\end{array}\right)
$$

the scalar mass matrix takes the form

$$
\begin{aligned}
& M^{2}=\mu_{0}^{2}+\alpha M_{0}+\beta M_{3}, \\
& M_{3}=N_{3}+N_{3}^{\dagger}, \quad \text { etc. },
\end{aligned}
$$

where for simplicity we take $\mu_{0}^{2}$ to be proportional to the unit matrix. The $M$ 's and their commutation relations with the generators of $T$ spin are given in appendix $\mathrm{B}$; $M_{0}$ is an isosinglet, and $M_{a}$ is an isovector.

By $U$ spin rotations we can write $\delta I I$ in the following convenient form:

$$
\delta \Pi=-\int \frac{\mathrm{d}^{4} k}{(2 \pi)^{4}} k^{2} \operatorname{Tr}\left[T \ldots \Delta_{1}\right]^{2}
$$

where

$$
\Delta_{1}^{-1}=k^{2}+\mu_{0}^{2}+\alpha M_{0}+\beta M_{1}
$$

The conjecture is that $\delta I I \geqslant 0$; we have been unable to establish this to all orders, but expanding in powers of $\alpha, \beta$ one finds a leading contribution

$$
\delta \mathrm{II}=\alpha^{2} \beta^{2} \int \frac{\mathrm{d}^{4} k}{(2 \pi)^{4}} \frac{k^{2}}{\left(k^{2}+\mu_{0}^{2}\right)^{6}} \operatorname{Tr}\left(M_{0}^{2} M_{3}^{2}\right),
$$

which is manifestly positive. We believe that this result is in fact true to all orders in $\alpha, \beta$ and continues to hold if we give distinct invariant masses to $\left(\chi . \xi_{1}, \xi_{2}\right)$.

At the end of sect. 3 it was shown that an arbitrary number of fermion doublets of the same hypercharge mixed with an arbitrary number of singlet fields gave $\delta \rho \geqslant 0$. Here we consider the generalization to the case in which one has more than one 
multiplet identical with respect to SU(2) but with different hypercharge. As will be seen below, such a model can yield either sign for $\delta \Pi$.

Consider the model described in sect. 2 consisting of two left doublets of different hypercharge and four right singlets [see eqs. (2.20) and (2.21)].

It is straightforward to apply our formalism to calculate $\delta \Pi$. If the left propagator is parametrized as

$$
\Delta_{\mathbf{L}}=\left(\begin{array}{cccc}
\boldsymbol{\alpha}_{+} & 0 & 0 & 0 \\
0 & \beta & \delta & 0 \\
0 & \delta & \gamma & 0 \\
0 & 0 & 0 & \alpha_{-}
\end{array}\right)
$$

then the integrand becomes

$$
\operatorname{Tr}\left\{-\frac{1}{2}\left[T_{+}, \Delta\right]\left[T_{-}, \Delta\right]+\left[T_{3}, \Delta\right]^{2}\right\}=\frac{1}{2}\left(\alpha_{-}-\beta\right)^{2}+\frac{1}{2}\left(\alpha_{-}-\gamma\right)^{2}-\delta^{2} .
$$

Clearly this will be negative if we can make $\alpha_{+} \approx \beta$ and $\alpha_{-} \approx \gamma$ for fixed $\delta$. But this can easily be arranged since $\alpha_{+}^{-1}=k^{2}+g_{a}^{2}$ and $\alpha_{-}^{-1}=k^{2}+g_{b}^{2}$, while $\beta, \gamma, \delta$ are independent of $g_{a}$ and $g_{b}$. However, one might worry that the integrand cannot be made negative for all momenta, so the integral for $\delta \Pi$ would turn out to be positive. In fact, it is possible to make the integrand negative definite, as can be seen by taking a special case, for example, $g_{b}^{\prime}=h, g_{a}^{\prime}=h^{\prime}=0, g_{a}=g_{b}$. In this case, one neutral particle is massless and the other has $m_{0}^{2}=2 h^{2}$. The two charged particles have been taken to have the same mass $m_{+}^{2}=m_{-}^{2}=g_{a}^{2}$, so this spectrum looks much like the preceding scalar example with negative $\delta$ II. Indeed, eq. (4.7) becomes simply

$$
\left(\frac{1}{k^{2}+m_{+}^{2}}-\frac{1}{k^{2}}\right)\left(\frac{1}{k^{2}+m_{+}^{2}}-\frac{1}{k^{2}+m_{0}^{2}}\right)=\frac{-m_{+}^{2}\left(m_{0}^{2}-m_{+}^{2}\right)}{\left(k^{2}+m_{+}^{2}\right)^{2} k^{2}\left(k^{2}+m_{0}^{2}\right)} .
$$

Thus, the integrand is negative definite whenever the charged particle mass lies between the two neutrals, just as before.

We now consider a fermion model which is $L R$ symmetric, i.e. has $T_{\mathrm{L}}=T_{\mathrm{R}}$. For such a model it is clear that gauge-invariant mass terms are allowed. as already discussed in the introduction in the context of the decoupling theorem.

Our model consists of a doublet $\left(\begin{array}{l}F_{1} \\ F_{2}\end{array}\right)_{\mathrm{L}+\mathrm{R}}$ which is coupled via Yukawa interactions to singlets $f_{1(\mathrm{~L}+\mathrm{R})}$ and $f_{2(\mathrm{~L}+\mathrm{R})}$. As usual a mass matrix is generated of the form

$$
\bar{\psi}_{L} m \psi_{\mathrm{R}}+\text { h.c. }
$$


where $\psi^{\mathrm{T}}=\left(F_{1} F_{2} f_{1} f_{2}\right)$ and $m$ has the general form

$$
\left(\begin{array}{cccc}
\mu & 0 & g_{1} & 0 \\
0 & \mu & 0 & g_{2} \\
g_{3} & 0 & \mu_{1} & 0 \\
0 & g_{4} & 0 & \mu_{2}
\end{array}\right),
$$

where $\mu_{,} \mu_{1}, \mu_{2}$ are gauge-invariant mass terms and $g_{1-4}$ arise from the Yukawa interactions. For general values of the $f$ 's it can be seen that $m$ breaks isospin like the third component of an isovector.

It follows easily that $\Delta_{L}$ and $\Delta_{R}$ are given by

$$
\Delta_{\mathrm{L}, \mathrm{R}}=\left(\begin{array}{cc}
A_{\mathrm{L}, \mathrm{R}} & B_{\mathrm{L}, \mathrm{R}} \\
B_{\mathrm{L}, \mathrm{R}} & D_{\mathrm{L}, \mathrm{R}}
\end{array}\right)
$$

in block form where the entries $A_{\mathrm{L}, \mathrm{R}}-D_{\mathrm{L}, \mathrm{R}}$ are diagonal matrices. The generators $T^{a}$ are given by

$$
T_{\mathrm{L}, \mathrm{R}}^{a}=\frac{1}{2}\left(\begin{array}{cc}
\tau^{a} & 0 \\
0 & 0
\end{array}\right)
$$

It is clear that in this model we will have contributions from both terms in eq. (3.21).

It is easy to show that

$$
\begin{aligned}
\operatorname{Tr}\left[T^{a}, \Delta\right]\left[T^{a}, \Delta\right] & \left.=\frac{1}{4} \operatorname{Tr}\left[\tau^{a}, A_{\mathrm{L}}\right]^{2}+\mathrm{L} \rightarrow \mathrm{R}, \quad \text { (no sum on } a\right), \\
2 \operatorname{Tr}\left[T^{a}, M \Delta\right]^{2} & =4 \operatorname{Tr} m \Delta_{\mathrm{R}} T^{a} m^{\dagger} \Delta_{\mathrm{L}} T^{a} \\
& =\operatorname{Tr}\left[\tau^{a}, E\right]^{2},
\end{aligned}
$$

where we have written $m \Delta_{\mathrm{R}}$ in block form:

$$
m \Delta_{\mathrm{R}}=\left(\begin{array}{cc}
E & F \\
G & H
\end{array}\right)
$$

(where the entries are again $2 \times 2$ diagonal matrices) and have dropped terms which cancel in the difference $\delta \rho$.

It is easy to see that both (4.12) and (4.13) give positive contributions to $\delta \rho$, since $A$ and $E$, both being diagonal matrices, can clearly be written as linear combinations of the unit matrix and $\tau_{3}$; whereupon the discussion following eq. (3.10) applies.

As already discussed in the introduction. one might wish to consider the limit in which some or all of the invariant mass terms $\left(\mu, \mu_{1}, \mu_{2}\right)$ become large. It is easy to 
show that in that limit the contributions to $\delta \rho$ from the heavy particles are of order $G_{\mathrm{F}} g^{4} / \mu^{2}$ so that the decoupling theorems hold as anticipated.

Up to this point we have not examined any fermion model containing representations of isospin greater than $\frac{1}{2}$. Therefore, as a final example we consider the case of a fermion representation whose left (right) handed components transform as a triplet and a singlet (two doublets). For simplicity we permit Yukawa interactions (with the Higgs) only between the triplet and the two doublets, so that the resulting mass spectrum consists of three massive and one massless field. If we write the mass term as $\bar{\psi}_{L} m \psi_{R}$, then

$$
m=\left(\begin{array}{cccc}
\sqrt{2} h_{1} & 0 & 0 & 0 \\
0 & h_{1} & h_{2} & 0 \\
0 & 0 & 0 & \sqrt{2} h_{2} \\
0 & 0 & 0 & 0
\end{array}\right)
$$

whence

$$
\begin{aligned}
& \Delta_{\mathrm{L}}=\left(k^{2}+m m^{\dagger}\right)^{\cdots 1} \\
& =\left|\begin{array}{llll}
\left(k^{2}+2 h_{1}^{2}\right)^{-1} & & & \\
& \left(k^{2}+h_{1}^{2}+h_{2}^{2}\right)^{-1} & & \\
& & \left(k^{2}+2 h_{2}^{2}\right)^{-1} & \\
& & & \left(k^{2}\right)^{-1}
\end{array}\right|, \\
& \Delta_{\mathrm{R}}=\left(k^{2}+m^{\dagger} m\right)^{-1}=\left(\begin{array}{cccc}
\left(k^{2}+2 h_{1}^{2}\right)^{-1} & 0 & 0 & 0 \\
0 & \frac{k^{2}+h_{2}^{2}}{\xi} & \frac{-h_{1} h_{2}}{\xi} & 0 \\
0 & \frac{-h_{1} h_{2}}{\xi} & \frac{k^{2}+h_{1}^{2}}{\xi} & 0 \\
0 & 0 & 0 & \left(k^{2}+2 h_{2}^{2}\right)^{-1}
\end{array}\right) \text {, }
\end{aligned}
$$

where

$$
\xi=k^{2}\left(k^{2}+h_{1}^{2}+h_{2}^{2}\right)
$$


As for the preceding case, in this model we find contributions from both terms in (3.21). A straightforward calculation gives

$$
\begin{aligned}
\operatorname{Tr}\left[T_{3}, \Delta\right]^{2}-\operatorname{Tr}\left[T_{+}, \Delta\right]\left[T_{-}, \Delta\right] & =\left\{\left(k^{2}+2 h_{1}^{2}\right)^{-1}-\left(k^{2}+h_{1}^{2}+h_{2}^{2}\right)^{-1}\right\}^{2} \\
+ & \left\{\left(k^{2}+2 h_{2}^{2}\right)^{-1}-\left(k^{2}+h_{1}^{2}+h_{2}^{2}\right)^{-1}\right\}^{2} \\
+ & \frac{1}{2}\left\{\left(k^{2}+2 h_{1}^{2}\right)^{-1}-\left(k^{2}+2 h_{2}^{2}\right)^{-1}+\left(h_{1}^{2}-h_{2}^{2}\right) / \xi\right\}^{2},
\end{aligned}
$$

$$
\begin{aligned}
\operatorname{Tr}\left[T_{3}, M \Delta\right]^{2}-\operatorname{Tr}\left[T_{+}, M \Delta\right]\left[T_{-}, M \Delta\right]= & \frac{\left(h_{1}^{2}+h_{2}^{2}\right)\left(h_{1}^{2}-h_{2}^{2}\right)^{2}}{\left(k^{2}+h_{1}^{2}+h_{2}^{2}\right)^{2}} \\
& \times\left\{\frac{1}{\left(k^{2}+2 h_{1}^{2}\right)^{2}}+\frac{1}{\left(k^{2}+2 h_{2}^{2}\right)^{2}}\right\}
\end{aligned}
$$

Thus both terms are separately non-negative, and vanish in the isospin symmetric limit $\left(h_{1}=h_{2}\right)$. We note once again the advantage of our formalism and the momentum-space representation in establishing this result.

From sect. 2 it can be seen that the mass matrix of this model continues to transform like the third component of an isovector if Yukawa interactions are also permitted between the singlet and the doublet fields. In that case we have shown that the isospin symmetric limit $\delta \rho=0$ is an extremum of $\delta \rho$. Of course, according to our conjectured theorem it is, in fact, the absolute minimum; although we have not demonstrated this explicitly we are confident that it is true.

A natural generalization of this model would be to the case of left-handed fields of $T$-spin $(J, J-1)$ and right-handed fields of $T$-spin $\left(J-\frac{1}{2}, J-\frac{1}{2}\right)$, but we have not carried out this calculation.

\section{Conclusions}

In this paper, we have investigated the one-loop contributions to $\rho$ due to heavy scalars and fermions. We have stressed that the isospin transformation properties of the mass matrix are important to determining the sign of the deviation of $\rho$ from one.

We have given a general classification of scalar and fermion contributions to $\rho$ at the one-loop level, and formulated a conjecture about the necessary and sufficient conditions for these radiative corrections $\delta \rho$ to be non-negative for all values of the 
parameters of the mass matrix. The class of models encompassed includes the $N$-generation generalization of the standard model of leptons and fermions, for which we have given a separate proof that $\delta \rho \geqslant 0$. We have also developed an alternative formalism for the calculation of $\delta \rho$.

At the one-loop level, non-zero $\delta \rho$ arises from isospin breaking in the mass matrix of the fermion or scalar fields. Clearly an independent source of such breaking arises from the coupling of the fields to the hypercharge current. This will contribute at the two-loop level; a naive estimate gives $\delta \rho \sim G_{\mathrm{F}} \alpha m^{2} /\left(8 \pi^{2}\right)$ which, while possibly as large as the one-loop corrections due to mass splitting, is phenomenologically negligible.

Within the next few years it is anticipated that precise measurements concerning $\mathrm{W}$ and $\mathrm{Z}$ masses will be made. One-loop calculations of the effect on these masses of normal generations of quarks and leptons have already been done [4]. It is evidently important to extend the considerations of this paper to $q^{2} \sim M_{\mathrm{W}}^{2}, M_{\mathrm{Z}}^{2}$; the precise values of $M_{\mathrm{w}}$ and $M_{\mathrm{Z}}$ will be a crucial test of the whole structure of the model. In the meantime, however, the measurement of $\rho$ remains our best 'window' on unexplored energy scales.

\section{Appendix A}

In this appendix, we derive some simple identities which result from the reparameterization invariance of one-loop integrals. The sort of expression with which we have been dealing takes the form [recall eq. (3.18)]

$$
\begin{aligned}
F\left(q^{2}\right) & =\int \mathrm{d}^{n} k \operatorname{Tr}\left[V_{1} S_{1}\left(k_{-}\right) V_{2} S_{2}\left(k_{-}\right)\right], \\
k_{\dot{-}} & =k+\left(\xi \pm \frac{1}{2}\right) q .
\end{aligned}
$$

For simplicity, we suppose the vertices $V_{i}$ are momentum independent. The $S_{i}(k)$ represent propagators, either boson or fermion, (and the trace is over all fields). Then, since $F\left(q^{2}\right)$ is independent of $\xi$, one can easily generate identities by taking derivatives with respect to $\xi$ :

$$
\begin{aligned}
& \frac{\partial F}{\partial \xi}=0=q_{\mu} \int \mathrm{d}^{n} k \operatorname{Tr}[\left.V_{1} \frac{\partial S_{1}\left(k_{+}\right)}{\partial k_{\mu}} V_{2} S_{2}\left(k_{-}\right)+V_{1} S_{1}\left(k_{+}\right) V_{2} \frac{\partial S_{2}\left(k_{-}\right)}{\partial k_{\mu}}\right] \\
& \frac{\partial^{2} F}{\partial \xi^{2}}=0=q_{\mu} q_{\nu} \int \mathrm{d}^{n} k \operatorname{Tr}\left[2 V_{1} \frac{\partial S_{1}\left(k_{+}\right)}{\partial k_{\mu}} V_{2} \frac{\partial S_{2}\left(k_{-}\right)}{\partial k_{\nu}}+V_{1} \frac{\partial^{2} S_{1}\left(k_{+}\right)}{\partial k_{\mu} \partial k_{\nu}} V_{2} S_{2}\left(k_{-}\right)\right. \\
&\left.+V_{1} S\left(k_{+}\right) V_{2} \frac{\partial^{2} S_{2}\left(k_{-}\right)}{\partial k_{\mu} \partial k_{\nu}}\right]
\end{aligned}
$$


In this paper, we have used these in the limit $q \rightarrow 0$ :

$$
\begin{gathered}
\int \mathrm{d}^{n} k \operatorname{Tr}\left[V_{1} \frac{\partial S_{1}}{\partial k_{\mu}} V_{2} S_{2}+V_{1} S_{1} V_{2} \frac{\partial S_{2}}{\partial k_{\mu}}\right]=0 \\
\int \mathrm{d}^{n} k \operatorname{Tr}\left[V_{1} \frac{\partial^{2} S}{\partial k_{g} \partial k_{\nu}} V_{2} S_{2}+V_{1} S_{1} V_{2} \frac{\partial^{2} S_{2}}{\partial k_{\mu} \partial k_{\nu}}\right]=-2 \int \mathrm{d}^{n} k \operatorname{Tr}\left[V_{1} \frac{\partial S_{1}}{\partial k_{\mu}} V_{2} \frac{\partial S_{2}}{\partial k_{\nu}}\right] .
\end{gathered}
$$

These are useful when expanding $F$ in powers of $q$. Alternatively, one may derive symmetric forms such as eq. (3.19) by shifting:

$$
\frac{\partial F}{\partial q_{\mu}}=\int \mathrm{d}^{n} k \operatorname{Tr}\left[V_{1} \frac{\partial S_{1}(k+1)}{\partial k_{\mu}} V_{2} S_{2}(k)\right] .
$$

Now shift $k \rightarrow k-q$ before taking the second derivative:

$$
\frac{\partial^{2} F}{\partial q_{\mu} \partial q_{\nu}}=-\int \mathrm{d}^{n} k \operatorname{Tr}\left[V_{1} \frac{\partial S_{1}(k)}{\partial k_{\mu}} V_{2} \frac{\partial S_{2}(k-q)}{\partial k_{\mu}}\right] .
$$

etc.

\section{Appendix B}

Because the Higgs field is an isodoublet, the weak isospin transformation properties of the mass terms are simple. Let $M$ be the mass matrix arising from spontaneous breakdown of trilinear (Yukawa) couplings. Define

$$
M_{\hat{n}}(\theta)=\mathrm{e}^{i \theta \hat{n} \cdot T} M \mathrm{e}^{-i \theta \hat{n} \cdot T} .
$$

Then it is easy to show that

$$
\frac{\mathrm{d}^{2} M_{\hat{n}}(\theta)}{\mathrm{d} \theta^{2}}=-\frac{1}{4} M_{\hat{n}}(\theta),
$$

so that

$$
M_{\hat{n}}(\theta)=M \cos \frac{1}{2} \theta+2 \sin \frac{1}{2} \theta \delta \mathscr{L}^{\hat{n}},
$$

where

$$
\delta \mathfrak{L}^{\hat{n}}=i[\hat{n} \cdot T, M] .
$$


One can use this to generate interesting identities, for example, the diagonal elements in eq. (3.19) are

$$
\Pi_{a a}(0)=-\frac{1}{8} \int \frac{\mathrm{d}^{n} k}{(2 \pi)^{n}} \operatorname{Tr}\left[\frac{\partial S_{a}}{\partial \theta} \gamma_{\mu} \frac{\partial S_{a}}{\partial \theta} \gamma_{\mu}\right],
$$

where

$$
S_{a}(\theta)=\mathrm{e}^{i \theta T_{a}} S \mathrm{e}^{-i \theta T_{u}},
$$

Now observe that the trace is in fact independent of $\theta$, so one need not set $\theta=0$. This leads to the amusing observation that (setting $\theta=\pi$ ), these expressions are invariant under the interchange

$$
M \leftrightarrow 2 \delta E_{a} .
$$

It is also easy to exploit the doublet character of the Higgs field to work out the general form for $\delta \ell_{a}$ directly from $M$, without actually writing down the generators $T_{a}$ and calculating the commutator $i\left[T_{a}, M\right]$. For example, suppose the Yukawa terms are of the form given in eq. (2.18) of sect. 2. Define the isoscalar $N_{0}=$ $\psi_{j_{1} \ldots j_{n}} X_{j_{1}, j_{2} \ldots j_{n}}$ and the isovectors $N_{a}=\psi_{j_{1} \ldots j_{n}}\left(\sigma_{a}\right)_{j_{1}}^{k} X_{k, j_{2} \ldots j_{n}}$, so that the mass matrix is

$$
M=y N_{0}+\Delta y N_{3}+\text { h.c. }
$$

Then

$$
\begin{aligned}
& \delta E_{3}=i\left[T_{3}, M\right]=\frac{1}{2} i\left(\Delta y N_{0}+N_{3} y\right)+\text { h.c., } \\
& \delta E_{1}=\frac{1}{2}\left(i y N_{1}+\Delta y N_{2}\right)+\text { h.c., } \\
& \delta E_{2}=\frac{1}{2}\left(i y N_{2}-\Delta y N_{1}\right)+\text { h.c. }
\end{aligned}
$$

Alternatively, one may write

$$
\begin{aligned}
& \delta \mathscr{Q}_{+}=\frac{1}{2} i\left[(y-\Delta y) N_{+}-(y+\Delta y) N_{-}^{\dagger}\right], \\
& \delta \mathscr{L}_{-}=\frac{1}{2} i\left[(y+\Delta y) N_{-}-(y-\Delta y) N_{+}^{\dagger}\right] .
\end{aligned}
$$

Thus, in practice, it is trivial to write down the vertices $\delta \varrho_{a}$ without actually calculating the commutator $i\left[T_{a}, M\right]$. 


\section{References}

[1] S. Weinberg, Phys. Rev. D19 (1979) 1277;

L. Susskind, Phys. Rev. D20 (1979) 2619

[2] I. Liede and M. Roos, Nucl. Phys. B167 (1980) 397;

J.E. Kim, P. Langacker, M. Levine and H.H. Williams, Rev. Mod. Phys. 53 (1981) 211

[3] M. Veltman, Nucl. Phys. B123 (1977) 89

[4] F. Antonelli. M. Consoli and G. Corbo, Phys. Lett. 91B (1980) 90;

M. Veltman. Phys. Lett. 91B (1980) 95

[5] D. Toussaint, Phys. Rev. D18 (1978) 1626

[6] W.J. Marciano and A. Sirlin, Phys. Rev. D22 (1980) 2695

[7] M.S. Chanowitz. M.A. Furman and I. Hinchliffe, Phys. Lett. 78B (1978) 285; Nucl. Phys. B153 (1978) 402

[8] M. Green and M. Veltman, Nucl. Phys. B169 (1980) 137; (E:BI75 (1980) 547)

[9] K. Lane and M. Peskin, Proc. Moriond Conf., 1980, vol. 2, p. 469

[10] T. Appelquist and J. Carazzone, Phys. Rev. DII (1975) 2856;

K. Symanzik, Comm. Math. Phys. 34 (1973) 7

[11] C. Senjanovic and A. Sokorac. Nucl. Phys. BI64 (1980) 305;

M. Yoshimura, Prog. Theor. Phys. 64 (1980) 353, 594:

Y. Kazama and Y.-P. Yao, Fermilab-Pub-81/18-THY (Jan., 1981);

Y. Kazama, D. Unger and Y.-P. Yao, University of Michigan UM-HE 80-36 (1981)

[12] T. Appelquist and R. Shankar, Nucl. Phys. B158 (1979) 317:

T. Appelquist and C. Bernard, Phys. Rev. D22 (1980) 200;

A.C. Longhitano, Phys. Rev. D22 (1980) 1166: Yale University preprint YTP-80-26, Nucl. Phys. B $188(1981) 118$

[13] S. Adler and R. Dashen. Current algebra (Benjamin. New York. 1968)

[14] A. Sirlin, Rev. Mod. Phys. D20 (1979) 274

[15] R. Dashen. Phys. Rev. D3 (1971) 1879 